\title{
Molecular characterization of bovine noroviruses and neboviruses in Turkey: detection of recombinant strains
}

\author{
Ilke Karayel-Hacioglu' ${ }^{1}\left[\right.$. Feray Alkan ${ }^{1}$
}

Received: 20 November 2018 / Accepted: 25 January 2019 / Published online: 8 March 2019

(c) Springer-Verlag GmbH Austria, part of Springer Nature 2019

\begin{abstract}
To investigate the molecular epidemiology and genetic diversity of bovine enteric caliciviruses, a total of 167 fecal samples from diarrheic calves were screened. Bovine noroviruses (BoNoVs) and neboviruses were detected in 56 (33.5\%) and 37 (22.1\%) fecal samples, respectively. Sequences of the RdRp and capsid gene of selected BoNoVs showed that the GIII.1 and GIII.2 genotypes were in circulation in Turkey. Two of the BoNoV strains were identified as recombinant strains (GIII. P1/GIII.2). All examined neboviruses possessed a Nebraska-like RdRp gene. The two nebovirus strains were classified into lineage 4 based on phylogenetic analysis of VP1 amino acid sequences. One of them showed evidence of a recombination event within the $\mathrm{S}$ domain. This study is thus the first to reveal the presence of the BoNoV GIII.1 genotype and recombinant strains of BoNoV and neboviruses in Turkey.
\end{abstract}

Caliciviruses are small non-enveloped viruses with a singlestranded, positive-sense RNA genome of 7.4 to $8.3 \mathrm{~kb}$. The family Caliciviridae consists of five genera, namely Vesivirus, Lagovirus, Norovirus, Sapovirus and Nebovirus, of which only noroviruses of genogroup III and neboviruses have been associated with calf diarrhea [1,2].

Based on the amino acid sequence of the complete VP1 capsid protein, noroviruses are divided into seven genogroups (GI to GVII) [3]. All bovine noroviruses (BoNoVs) belong to GIII, which includes three distinct genotypes [4, 5]. Two genotypes, GIII.1 and GIII.2, have been linked to calf diarrhea, represented by prototype strains Bo/Jena/80/ $\mathrm{DE}$ and Bo/Newbury2/76/UK, respectively [6, 7]. Additionally, a strain detected in sheep in New Zealand has been proposed to represent a third genotype, GIII.3 [5].

Neboviruses were first described as a genus of the family Caliciviridae in 2010 [8]. Based on analysis of the RdRp gene, three genotypes have been suggested for this genus,

Handling Editor: Reimar Johne.

Electronic supplementary material The online version of this article (https://doi.org/10.1007/s00705-019-04186-0) contains supplementary material, which is available to authorized users.

Ilke Karayel-Hacioglu

ilkeekarayel@gmail.com

1 Department of Virology, Ankara University, Faculty of Veterinary Medicine, Diskapi, 06110 Ankara, Turkey with the following prototypes: Bo/Newbury1/76/UK, Bo/ Nebraska/80/US and Bo/DijonA216/06/FR [1, 6, 9]. The capsid gene of neboviruses can be divided into two genotypes. One genotype consists of strains Bo/LZB-1/17/CH and Bo/DijonA216/06/FR, and the other can be divided into four capsid lineages [9-11].

Recombination within the same genogroup or genotype, or between genogroups occurs frequently in caliciviruses, and this is recognized as an important mechanism for its continual emergence [12]. Recombination events in the norovirus genotype usually occur close to or within the ORF1/ ORF2 junction [13]. Molecular studies have revealed that the BoNoV recombinant types represent two RdRp/capsid combinations, GIII.P1/GIII.2 and GIII.P2/GIII.1 [13]. The first recombinant BoNoV strain, Bo/Thirsk10/00/UK, had a GIII.1-related RdRp but a GIII.2-related capsid [14]. Molecular investigations of isolates from various geographical regions have shown that most naturally occurring BoNoV recombinants share the same combination with Bo/Thirsk10/00/UK [12, 15], whereas only one recombinant strain with a GIII.P2/GIII.1 combination has been described to date [13]. Recombination events can also occur in neboviruses. Indeed, two recombinant nebovirus strains (Bo/16TE/2010/ITA and Bo/LZB-1/17/CH) have been identified in recent studies $[11,16]$.

Since bovine enteric caliciviruses (BECs) are routinely excluded from diagnostic procedures for identifying enteric diseases, their real impact on livestock production is unclear. 
Unlike other enteric viruses, such as bovine rotavirus group A (BRVA), and bovine coronavirus (BCoV), there is limited knowledge of BECs in Turkey [17-20]. Before the conception of this study, all reports on BEC infections only addressed the detection of BoNoVs in fecal samples from diarrheic calves [19]. Thus, to investigate the molecular epidemiology and genetic diversity of BECs, a total of 167 fecal samples from diarrheic calves (ages 1 day to 7 months) were collected from 48 family farms (up to 10 cattle) and 11 organized dairy cattle farms in 10 different provinces of Turkey between 2002 and 2016 (Table S1 and Table S2).

Coinfection is frequently observed in diarrheic calves, although a single primary pathogen can be the cause in some cases. Therefore, the fecal samples were also screened for other enteric pathogens frequently detected in Turkey, such as BRVA, BCoV, E. coli and Cryptosporidium spp., using a commercial ELISA kit (Bio-X Diagnostics S.A., Belgium) according to the manufacturer's instructions.

Viral RNA was extracted from $250 \mu \mathrm{l}$ of fecal suspension $(1: 10, w / v)$ using the TRIzol LS procedure. All extracts were stored at $-80^{\circ} \mathrm{C}$ until tested. Reverse transcription was performed using a RevertAid First Strand cDNA Synthesis Kit (Thermo Fisher Scientific, USA) according to the manufacturer's instructions. BECs were detected by nested RTPCR using two sets of external and internal primers targeting the RdRp gene. The following primer sets were used: for BoNoVs, CBECU-F/R (532 bp) and nCBECU-F/R (326 bp); for neboviruses, NBU-F/R (549 bp) and nNBU-F/R (194 bp) [21, 22]. Nested RT-PCR was performed as described previously $[21,22]$. The capsid gene of the BoNoVs strains was amplified using two primer sets (NAcap-F/R and nCBECU-F/BoNoVcap-A) [12, 15, 22]. The primers NAcap-F/R (1615 bp) targeted the full-length capsid gene of BoNoVs, while the primer set nCBECU-F/BoNoVcap-A (781 bp) was used to amplify the $3^{\prime}$ end of RdRp and the 5' end of the capsid gene. The primer set used for the amplification of the full-length nebovirus capsid gene (1692 bp) was NBcap-F3/R [12]. RT-PCR was performed using a Phusion High-Fidelity PCR Kit (Thermo Fisher Scientific, USA) as described previously $[12,15]$ with some modifications. The amplification products were analyzed by $1 \%$ agarose gel electrophoresis and visualized under UV light.

Some PCR products with the expected size were selected for sequencing based on the sampling year and the location. Purified PCR products were sequenced in both directions with the same primers used for amplification. Cognate sequences of reference BoNoVs and neboviruses representing different genotypes were retrieved from the GenBank database using the BLAST engine. Multiple sequence alignments were prepared by the MUSCLE algorithm as implemented in Aliview Software [23, 24]. MEGA 7.0 was used to build phylogenetic trees [25], the statistical significance of which was estimated by bootstrap analysis (1000 replicates).
Nucleotide (nt) and amino acid (aa) identity values were calculated in CLC Main Workbench (CLC Bio-QIAGEN, Aarhus, Denmark). Genetic recombination was analyzed using SimPlot software (version 3.5.1). All sequences ( $>200 \mathrm{bp}$ ) detected in this study have been made available in the GenBank database under the following accession numbers: KX184721-KX184724; KX216596, KX216597; KX189067-KX189100.

Among the 167 samples from diarrheic calves, BECs were detected in $76(45.5 \%)$. BoNoVs and neboviruses were detected in $56(33.5 \%)$ and 37 (22.1\%) fecal samples, respectively. Coinfection with BoNoVs and neboviruses was detected in $17(10.1 \%)$ samples. BECs were detected on 20 family farms and 10 commercial dairy cattle farms, with 15 family farms and 8 commercial farms positive for BoNoVs, and 11 family farms and 7 commercial farms positive for neboviruses (Table S1 and Table S2). The results from the selected representatives for different farms and geographical regions showed that BECs are prevalent in Turkey (Table S3 and Table S4). Out of 76 BEC-positive samples, 18 and 14 were positive for the $\mathrm{BoNoV}$ and nebovirus alone, respectively, while the rest of the 44 fecal samples tested positive for other enteric pathogens. Of the concurrent infections of BECs with other pathogens, Cryptosporidium spp. was most commonly seen (46.5\%), followed by BRVA (31.9\%) and $\mathrm{BCoV}(20.8 \%)$. However, no $E$. coli was detected in any of the samples.

Molecular analysis of the partial RdRp gene sequences obtained from the selected $34 \mathrm{BoNoV}$ strains revealed that nine BoNoV strains were clustered in GIII.1, whereas the remaining 25 BoNoV strains were located in GIII.2 (Fig. 1A). The sequenced samples shared $69.5-100 \% \mathrm{nt}$ sequence identity with each other. The nine BoNoV strains clustered in GIII.1 displayed 81.4-90.2\% nt sequence identity with Bo/Jena/80/DE, while the remaining $25 \mathrm{BoNoV}$ strains clustered in GIII.2 displayed 84.2-91.9\% nt sequence identity with Bo/Newbury2/76/UK. Molecular analysis of the partial RdRp gene sequences demonstrated that the 20 nebovirus strains displayed $86.3-100 \%$ nt sequence identity with each other, and the phylogenetic tree confirmed that they are more closely related to Bo/Nebraska/80/US (82.4-87\% nt) than to Bo/Newbury1/76/UK (75.3-80.5\% nt) (Fig. 1B).

Considering that recombination frequently occurs at the ORF1-ORF2 junction of noroviruses, the capsid gene was amplified. Amplicons of the expected size for the full-length capsid gene for four strains (Aksaray/K59, Aksaray/K063, Aksaray/K056, and Aksaray/K060) and a partial capsid gene for one (Aksaray/K066) were successfully obtained. The sequences of all full-length capsid genes shared higher $\mathrm{nt}$ and aa sequence identity with Bo/Newbury2/76/UK (82.8$88.5 \%$ nt, 91.1-96.9\% aa) than with Bo/Jena/80/DE (64.8$68.5 \% \mathrm{nt}$ and $66.6-69.7 \%$ aa). Looking at the phylogenetic 


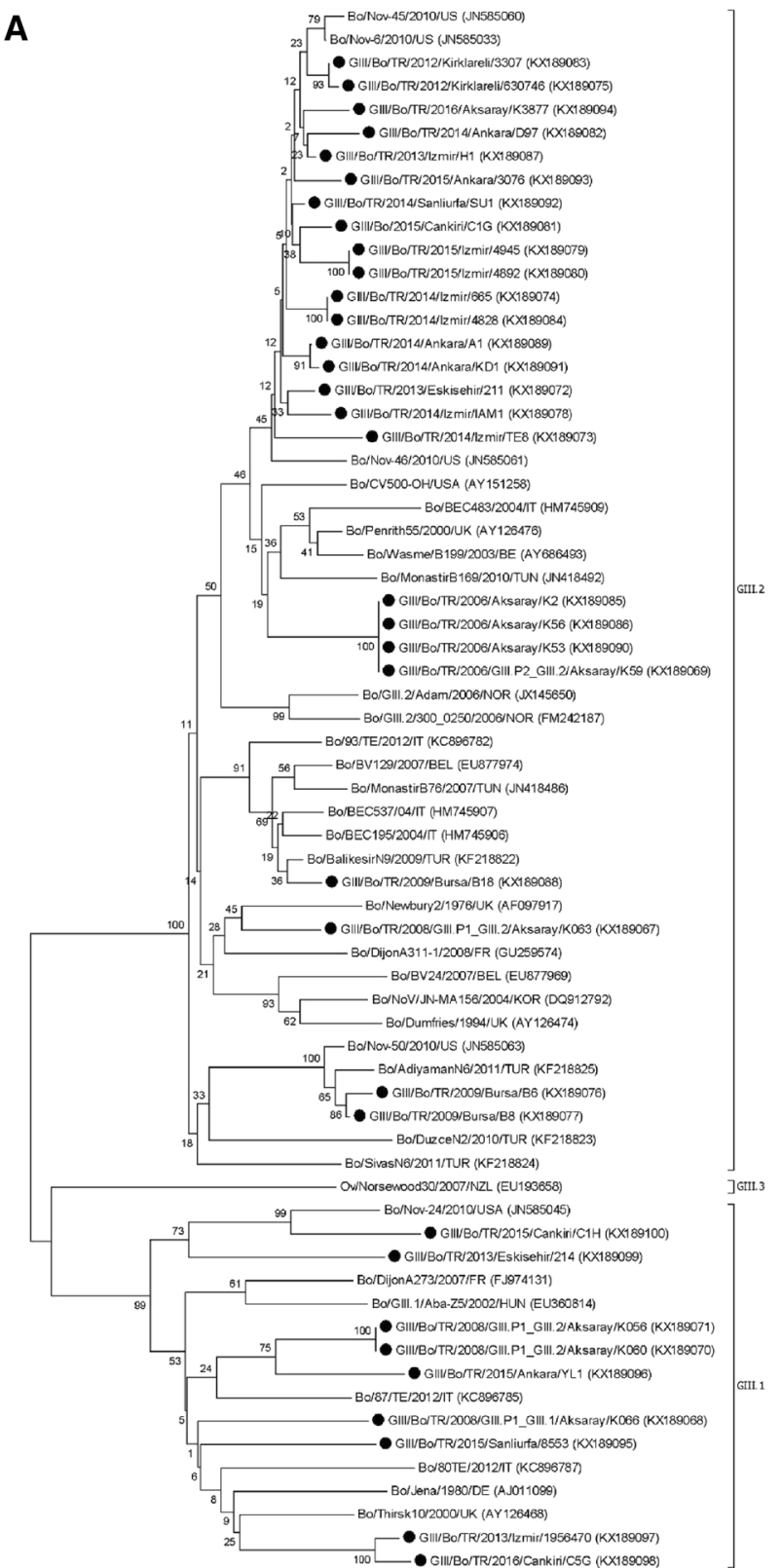

$\longdiv { 0 . 0 2 }$

Fig. 1 Phylogenetic trees based on the partial nucleotide sequences of the RdRp genes of A) BoNoVs (326 bp) and B) neboviruses (194 bp). The phylogenetic trees were constructed using the neighbor-joining method and the p-distance nucleotide substitution model in MEGA 7.

analysis, these four sequences were more closely related to GIII.2 (Fig. 2A), whereas Aksaray/K066 was clustered in GIII.1 (Fig. S1).

Based on the phylogenetic analysis (ORF1/RdRp and ORF2/Capsid), the conflicting results obtained in grouping the RdRp and capsid genes of Aksaray/K056 and Aksaray/ K060 strains may indicate recombination within the ORF1 and ORF2 junction. Accordingly, both strains displayed the

\section{B}

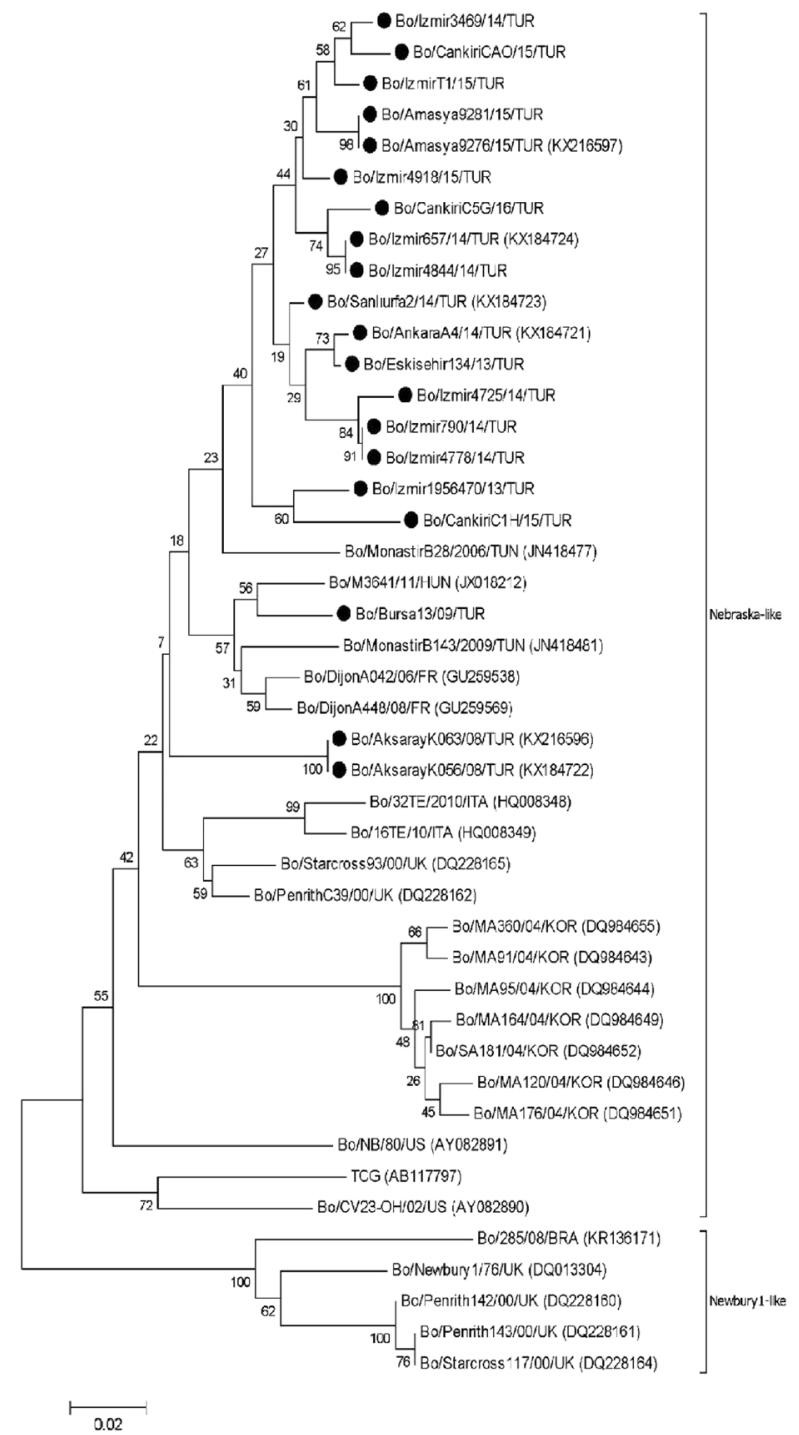

Statistical significance was estimated by bootstrap analysis (1000 replicates). The strains investigated in this study are indicated by black dots

same RdRp type as GIII.1, whereas their capsid types were genetically closest to GIII.2. Based on a SimPlot analysis performed on the sequence of the partial ORF1 and the fulllength ORF2, a putative recombination breakpoint located within the ORF1/ORF2 junction (nt position 327) was identified (Fig. 2B).

For neboviruses, a full-length capsid gene for two strains was successfully recovered (AksarayK056 and 
AnkaraA4). These strains showed $87.4 \%$ nt and $96.1 \%$ aa sequence identity to each other. Regarding the reference virus strains of each nebovirus genotype, AksarayK056 and AnkaraA4 shared $85.4 \%$ and $83.8 \%$ nt $(92.9 \%$ and $91.6 \%$ aa) sequence identity with Bo/Newbury $1 / 76 / \mathrm{UK}$ and $86.5 \%$ and $85.4 \%$ nt (93\% and $91.9 \%$ aa) sequence identity with Bo/Nebraska/80/US, respectively. Based on phylogenetic analysis, AksarayK056 and AnkaraA4 were clustered in lineage 4. AksarayK056 formed a separate branch with Bo/Dijon058/FR, while AnkaraA4 was grouped with the strain Bo/DijonA386/FR and the recombinant strain Bo/16TE/2010/ITA (Fig. 3A). The two strains with a lineage 4 capsid type, both of which were related to reference virus Bo/Nebraska/80/US (lineage 1) based on the RdRp gene, were further analyzed for possible recombination events. Recombination analysis did not show any recombination events in the genome of AksarayK056. Strain AnkaraA4 closely resembled Nebraska in RdRp but resembled Bo/Penrith143/00/UK, Bo/16TE/2010/ITA and Bo/DijonA386/08/FR (lineage 4) in the capsid region. The UK strain has a Newbury1-like RdRp, while Bo/16TE/2010/ ITA and Bo/DijonA386/08/FR have a Nebraska-like RdRp. Accordingly, AnkaraA4 was a possible recombinant strain, and a putative recombination breakpoint was identified within the $\mathrm{S}$ domain of the capsid protein (Fig. 3B).

The involvement of BECs in calf diarrhea has become an important issue in recent years, and the prevalence of these viruses has therefore been investigated in many countries. In this study, we identified BoNoV (33.5\%) and nebovirus (22.1\%) infections in diarrheic calves from 48 family farms and 11 commercial dairy cattle farms in 10 different provinces of Turkey. Surprisingly, BECs were detected on commercial dairy farms at a much higher rate than on family farms. This could be due to the large number of animals on commercial farms and/or routine vaccination against the other enteropathogens. Further epidemiological studies with
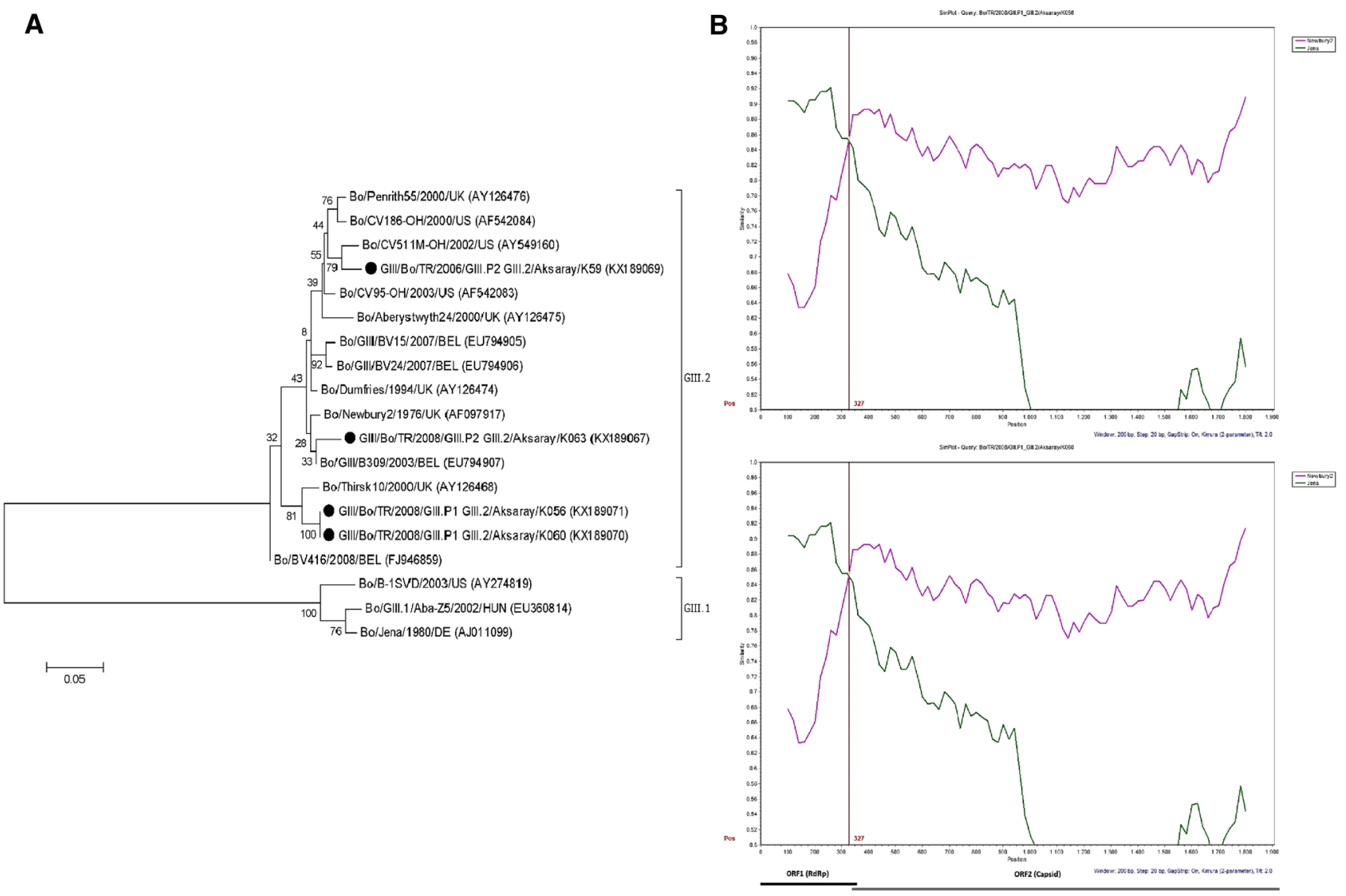

Fig. 2 A) BoNoV phylogenetic tree based on the deduced 522-aa sequences of the complete capsid (VP1). The tree was constructed by the maximum-likelihood method with bootstrap values calculated for 1000 replicates. The scale bar indicates amino acid substitutions per site. The strains investigated in this study are indicated by black dots. B) SimPlot analysis of Aksaray/K056 and Aksaray/K060 strains. A nucleotide identity plot comparing the genomes of the Aksaray/K056

and Aksaray/K060 strains (bp 1-342 belonging to RdRp and bp 3431908 belonging to the complete capsid) with Bo/Jena/80/DE and Bo/ Newbury2/76/UK is shown. The putative recombination sites of both strains (nt position 327) are located within the ORF1/ORF2 junction. SimPlot analysis was performed using a window size of $200 \mathrm{nt}$ and a step size of $20 \mathrm{nt}$ 
A

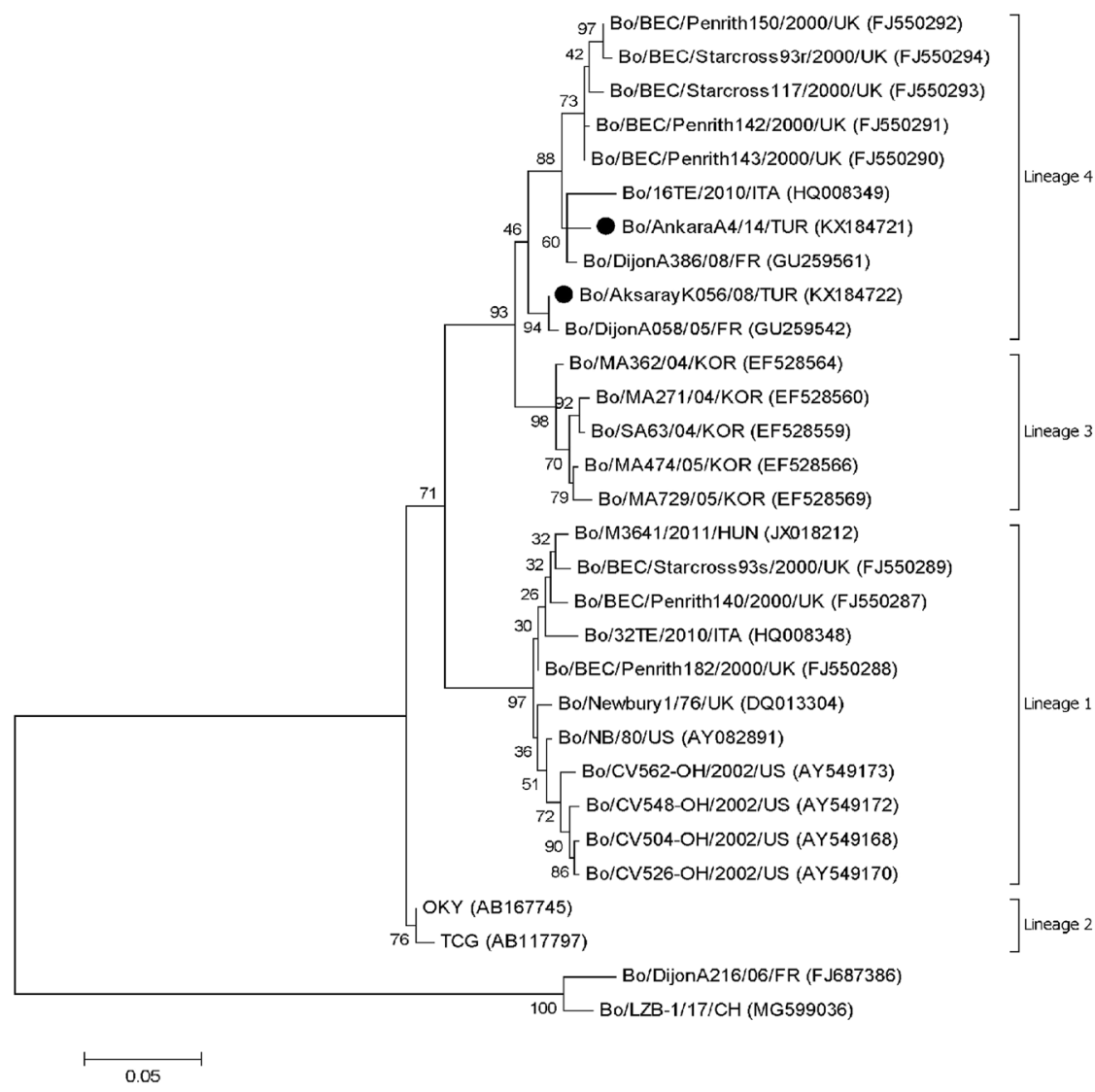

B

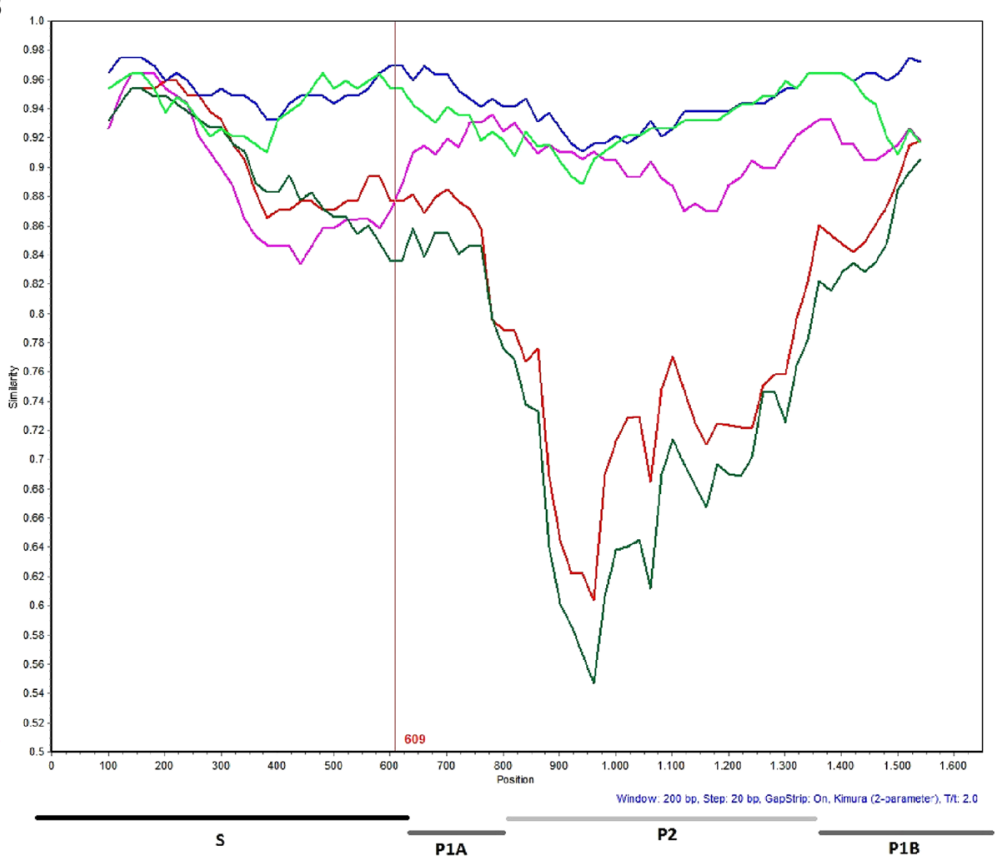

Fig. 3 A) Nebovirus phylogenetic tree based on the deduced 549-aa sequences of the complete major capsid (VP1). The tree was constructed by the maximum-likelihood method with bootstrap values calculated for 1000 replicates. The scale bar indicates amino acid substitutions per site. The strains investigated in this study are indicated by black dots. B) SimPlot analysis of strain AnkaraA4. The nucleotide identity plot comparing the genome sequence of the
AnkaraA4 strain with those of Bo/Penrith143/00/UK, Bo/16TE/2010/ ITA, Bo/Newbury1/76/UK, Bo/DijonA386/08/FR and Bo/ Nebraska/80/US nebovirus strains is shown. The capsid gene is divided into S, P1A, P1B and P2 domains. The putative recombination site of AnkaraA4 (nt position 609) is located at the end of the $\mathrm{S}$ domain. SimPlot analysis was performed using a window size of $200 \mathrm{nt}$ and step size of $20 \mathrm{nt}$ 
a larger number of samples would help to explain the differences in the detection rates between the two types of farms.

BoNoVs were detected in diarrheic calves at a much higher rate than previously seen in Turkish reports (1.78.5\%) [18-20] but at a similar rate to that reported in European countries and North America $[9,21,26]$. The prevalence rate of nebovirus was higher than the reported rates in Tunisia [27], Brazil [28], Italy [16], France [9], South Korea [29] and the United Kingdom [30], while it was similar to prevalence rates (21.6-39.5\%) reported in North America and Iran [21, 26, 31]. There has been only one other study on the prevalence of neboviruses (25.19\%) in Turkey [20], and the similarity in its reported prevalence rates to those seen here is noteworthy. The higher prevalence rate of $\mathrm{BoNoV}$ compared to that of nebovirus in this study was an expected observation, as other investigators previously reported this in Tunisia and France $[9,27]$. The results show that BECs are prevalent in Turkey and have been recorded in various cases of diarrheic calves, being commonly detected alone or in combination with other agents such as BRVA, BCoV and Cryptosporidium spp. The detected prevalence of BECs demonstrates their importance in calf diarrhea in Turkey, as in other countries $[9,15,26,27]$. Therefore, BECs should be included in the routine diagnostic procedure for calf diarrhea to reveal the real causative agent(s), to determine their contributions to clinical manifestations and to take precautions to prevent calf diarrhea in Turkey.

This study not only identified GIII.2, which had been documented previously [18-20], but was also able to identify the GIII.1 genotype for the first time ever in Turkey. Like this study, many studies in the USA, Europe, South Korea and Argentina have also detected both genotypes, although the GIII.2 genotype predominates [9, 16, 21, 32, 33]. Possible reasons for the low rate of detection of the GIII.1 genotype include the presence of GIII.P2/GIII.1 recombinant BoNoVs and the short duration of viral shedding of this genotype [2].

Another interesting result was the detection of two potential recombinant BoNoV strains (Aksaray/K056 and Aksaray/K060). They are the first recombinant BoNoV strains described in Turkey and were identified as GIII. P1/GIII.2, similar to other recombinant strains reported in Europe $[14,15]$. These recombinant strains were detected on the same farm (farm no. 2, Aksaray) sampled from diarrheic calves in 2008. Fecal samples were collected from this farm in three different years (2006, 2008 and 2016). BoNoVs were detected in each sampling year while genotypes GIII.2 were identified in 2006 and 2016, and GIII.1 and GIII.2 were identified in 2008. These results support the argument that the presence of different genotypes concurrently is an important factor in increasing recombination [12]. As in other studies $[13,15]$, these findings indicate that genotyping based only on the RdRp sequence may not be sufficient for characterizing BoNoVs due to the existence of a preferential recombination site in the ORF1-ORF2 junction region. Thus, sequence analysis of the capsid gene seems to be necessary for precise characterization $[13,15]$.

Molecular and phylogenetic analyses of a portion of the $\mathrm{RdRp}$ region of the neboviruses in this study characterized them as Nebraska-like, similar to those reported in the USA, Italy, South Korea, Tunisia and Turkey [16, 20, 21, 27, 29]. Although there are only a few studies on the epidemiology of neboviruses, the results obtained so far indicate that Newbury1-like strains have been detected only in the United Kingdom, Brazil and Iran [28, 30, 31].

Few studies have presented data about the capsid gene region of neboviruses. Our results show that nebovirus capsid lineage 4 is present in Turkey. AnkaraA4 is considered a potential recombinant strain due to the inconsistencies between RdRp and capsid clustering. To date, only two recombinant nebovirus strains have been reported, and their recombination sites are within the RdRp gene or at the end of the $\mathrm{S}$ domain $[11,16]$. This study revealed that the AnkaraA4 strain has undergone a recombination event, with the breakpoint located within the S domain of the capsid protein, which is similar to another recombinant nebovirus strain, Bo/16TE/2010/ITA [16]. These results are also consistent with previous studies, suggesting that molecular analysis of the full-length capsid gene region is required to determine nebovirus lineages [10].

In conclusion, the present study showed that both BoNoVs and neboviruses contribute to calf diarrhea, either alone or together with other enteropathogens. Genetic analysis revealed that both BoNoV GIII.1 and GIII.2 genotypes are circulating in Turkey, which allowed us to report the detection of GIII.1 in Turkey for the first time. This study also provides new genetic data for neboviruses in Turkey, which shows that they are genetically closely related to $\mathrm{Bo} /$ Nebraska/80/US in the RdRp region and have a lineage 4 capsid type. Furthermore, this study is the first to report the presence of recombinant strains of BoNoVs and neboviruses in Turkey. Large-scale epidemiological studies would enhance our understanding of the genetic characteristics and evolution of BECs. Finally, considering their contribution to calf diarrhea, BECs might be incorporated into cattle vaccines in the future.

Acknowledgements This manuscript represents a portion of a thesis submitted by IKH to the Department of Virology, Faculty of Veterinary Medicine, Ankara University, in fulfillment of the requirements for a $\mathrm{PhD}$ degree. We thank Dr. Krisztian Banyai for his support and advice as a supervisor during the Fellowship Program (2214A) at the Institute for Veterinary Medical Research, Centre for Agricultural Research, Hungarian Academy of Sciences, Budapest, Hungary.

Funding This project was funded by Ankara University Scientific Research Projects Coordination Unit (project no. 13L3338011). IKH was supported by the General National PhD Scholarship Program (2211A) and the International Doctoral Research Fellowship Program 
(2214A) of the Scientific and Technological Research Council of Turkey (TUBITAK).

\section{Compliance with ethical standards}

Conflict of interest The authors declare that they have no conflict of interest.

Ethical approval This article does not contain any studies with animals performed by any of the authors.

\section{References}

1. Smiley JR, Chang KO, Hayes J et al (2002) Characterization of an enteropathogenic bovine calicivirus representing a potentially new calicivirus genus. J Virol 76:10089-10098

2. Otto PH, Clarke IN, Lambden PR et al (2011) Infection of calves with bovine norovirus GIII.1 strain jena virus: an experimental model to study the pathogenesis of norovirus infection. J Virol 85:12013-12021

3. Vinjé $\mathbf{J}$ (2015) Advances in laboratory methods for detection and typing of norovirus. J Clin Microbiol 53:373-381

4. Oliver SL, Batten CA, Deng Y et al (2006) Genotype 1 and genotype 2 bovine noroviruses are antigenically distinct but share a cross-reactive epitope with human noroviruses. J Clin Microbiol 44:992-998

5. Wolf S, Williamson W, Hewitt J et al (2009) Molecular detection of norovirus in sheep and pigs in New Zealand farms. Vet Microbiol 133:184-189

6. Woode GN, Bridger JC (1978) Isolation of small viruses resembling astroviruses and caliciviruses from acute enteritis of calves. J Med Microbiol 11:441-452

7. Günther H, Otto P (1987) Diarrhea in young calves. 7. "Zackenvirus"(Jena agent 117/80)—a new diarrhea pathogen in calves. Arch Exp Veterinarmed 41:934

8. Carstens EB (2010) Ratification vote on taxonomic proposals to the International Committee on Taxonomy of Viruses (2009). Arch Virol 155:133-146

9. Kaplon J, Guenau E, Asdrubal P et al (2011) Possible novel nebovirus genotype in cattle, France. Emerg Infect Dis 17:1120-1123

10. D'Mello F, Jervis SM, Edwards PM et al (2009) Heterogeneity in the capsid protein of bovine enteric caliciviruses belonging to a new genus. Virology 387:109-116

11. Guo Z, He Q, Yue H et al (2018) Genomic characterization of a RdRp-recombinat nebovirus strain with a novel VP1 genotype. Virus Res 251:6-13

12. Han MG, Smiley JR, Thomas C, Saif LJ (2004) Genetic recombination between two genotypes of genogroup III bovine noroviruses (BoNVs) and capsid sequence diversity among BoNVs and Nebraska-like bovine enteric caliciviruses. J Clin Microbiol 42:5214-5224

13. Bull RA, Tanaka MM, White PA (2007) Norovirus recombination. J Gen Virol 88:3347-3359

14. Oliver SL, Brown DW, Green J, Bridger J (2004) A chimeric bovine enteric calicivirus: evidence for genomic recombination in genogroup III of the Norovirus genus of the Caliciviridae. Virology 326:231-239
15. Di Martino B, Di Profio F, Di Felice E et al (2014) Genetic heterogeneity of bovine noroviruses in Italy. Arch Virol 159:2717-2722

16. Di Martino B, Di Profio F, Martella V et al (2011) Evidence for recombination in neboviruses. Vet Microbiol 153:367-372

17. Alkan F, Karayel İ, Catella C et al (2015) Identification of a bovine enteric calicivirus, Kirklareli virus, distantly related to neboviruses, in calves with enteritis in Turkey. J Clin Microbiol 53:3614-3617

18. Gulacti I, Sozdutmaz I, Isıdan H (2016) Molecular characterization of the bovine noroviruses from diarrhoeic calves in Turkey 2. Turk J Vet Anim Sci 40:1-6

19. Yilmaz H, Turan N, Altan E et al (2011) First report on the phylogeny of bovine norovirus in Turkey. Arch Virol 156:143-147

20. Turan T, Işıdan H, Atasoy MO, Irehan B (2018) Detection and molecular analysis of bovine enteric norovirus and nebovirus in Turkey. J Vet Res 62:129-135

21. Smiley JR, Hoet AE, Traven M et al (2003) Reverse transcription-PCR assays for detection of bovine enteric caliciviruses (BEC) and analysis of the genetic relationships among BEC and human caliciviruses. J Clin Microbiol 41:3089-3099

22. Park SJ, Jeong C, Yoon SS et al (2006) Detection and characterization of bovine coronaviruses in fecal specimens of adult cattle with diarrhea during the warmer seasons. J Clin Microbiol 44:3178-3188

23. Larsson A (2014) AliView: a fast and lightweight alignment viewer and editor for large datasets. Bioinformatics 30:3276-3278

24. Edgar RC (2004) MUSCLE: multiple sequence alignment with high accuracy and high throughput. Nucleic Acids Res 32:1792-1797

25. Kumar S, Stecher G, Tamura K (2016) MEGA7: molecular evolutionary genetics analysis version 7.0 for bigger datasets. Mol Biol Evol 33:1870-1874

26. Cho YI, Han JI, Wang C et al (2013) Case-control study of microbiological etiology associated with calf diarrhea. Vet Microbiol 166:375-385

27. Hassine-Zaafrane M, Kaplon J, Sdiri-Loulizi K et al (2012) Molecular prevalence of bovine noroviruses and neboviruses detected in central-eastern Tunisia. Arch Virol 157:1599-1604

28. Candido M, Alencar ALF, Almeida-Queiroz SR et al (2016) First detection and molecular characterization of Nebovirus in Brazil. Epidemiol Infect 144:1876-1878

29. Park SI, Jeong C, Park SJ et al (2008) Molecular detection and characterization of unclassified bovine enteric caliciviruses in South Korea. Vet Microbiol 130:371-379

30. Oliver SL, Asobayire E, Dastjerdi AM, Bridger JC (2006) Genomic characterization of the unclassified bovine enteric virus Newbury agent-1 (Newbury1) endorses a new genus in the family Caliciviridae. Virology 350:240-250

31. Pourasgari F, Kaplon J, Sanchooli A et al (2018) Molecular prevalence of bovine noroviruses and neboviruses in newborn calves in Iran. Arch Virol 163:1271-1277

32. Ferragut F, Vega CG, Mauroy A et al (2016) Molecular detection of bovine Noroviruses in Argentinean dairy calves: circulation of a tentative new genotype. Infect Genet Evol 40:144-150

33. Park SI, Jeong C, Kim HH et al (2007) Molecular epidemiology of bovine noroviruses in South Korea. Vet Microbiol 124:125-133

Publisher's Note Springer Nature remains neutral with regard to jurisdictional claims in published maps and institutional affiliations. 